Supporting Information for

\title{
Self-Powered Water Flow-Triggered Piezocatalytic Generation of Reactive Oxygen Species for Water Purification in Simulated Water Drainage
}

Shenyu Lan,${ }^{\dagger}$ Chuan $\mathrm{Yu},{ }^{\dagger}$ Enya Wu,${ }^{\dagger}$ Mingshan Zhu, ${ }^{\dagger, *}$ Dionysios D. Dionysiou ${ }^{\ddagger},{ }^{*}$

$\dagger$ Guangdong Key Laboratory of Environmental Pollution and Health, School of Environment, Guangdong-Hongkong-Macau Joint Laboratory of Collaborative Innovation for Environmental Quality, Jinan University, Guangzhou 511443, P.R. China

$\$$ Environmental Engineering and Science Program, Department Chemical and Environmental Engineering, University of Cincinnati, Cincinnati, OH, 45221-0012, USA

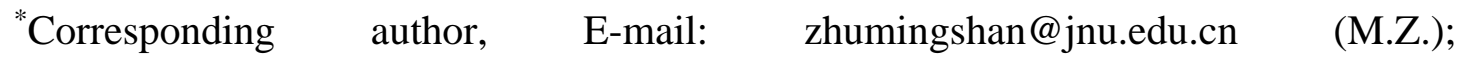
dionysios.d.dionysiou@uc.edu (D.D.D.) 


\section{Supporting Figures and Figure Caption}
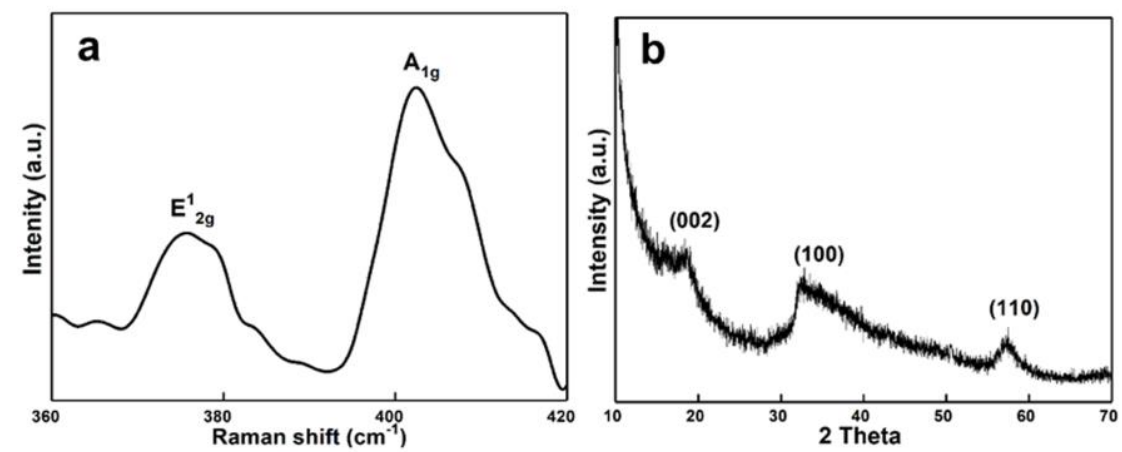

Figure S1. Raman spectrum (a) and XRD pattern (b) of $\mathrm{MoS}_{2}$.
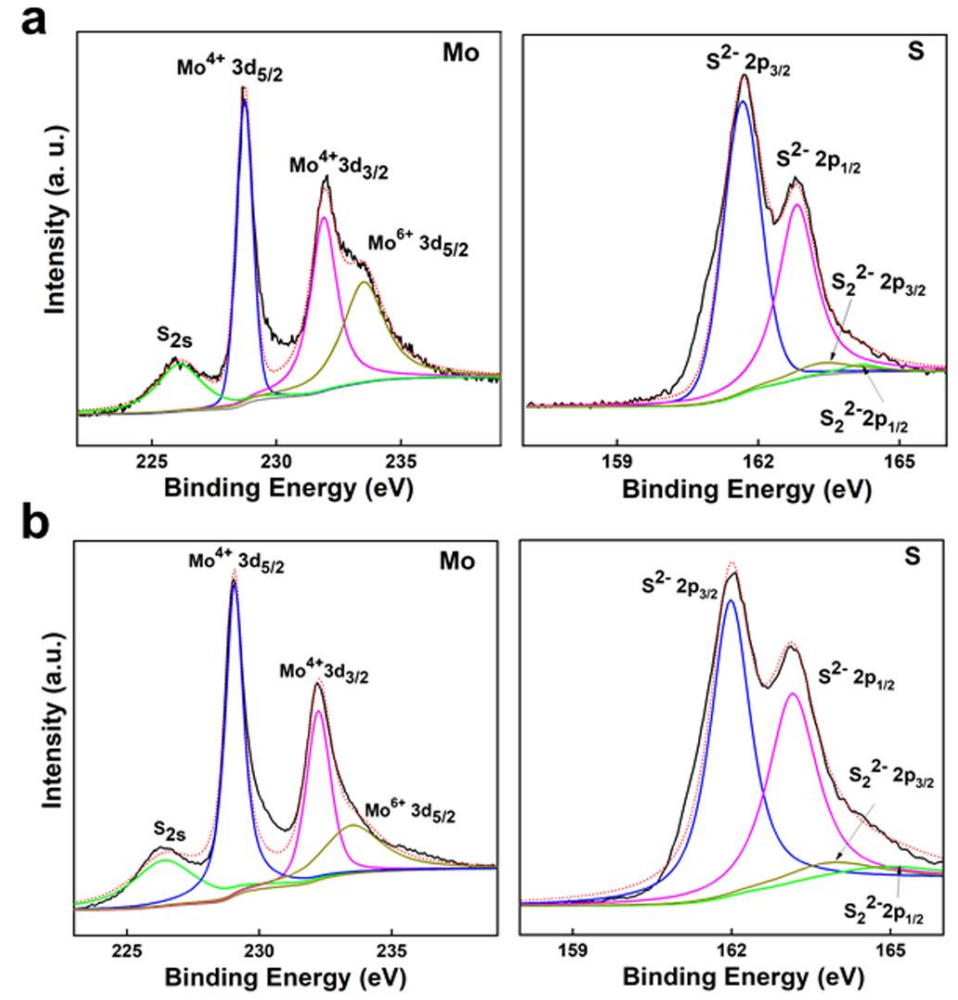

Figure S2. XPS spectra of Mo $3 d$ and S 2p for the fresh $\mathrm{MoS}_{2}$ (a) and the used $\mathrm{MoS}_{2}$ (b). 


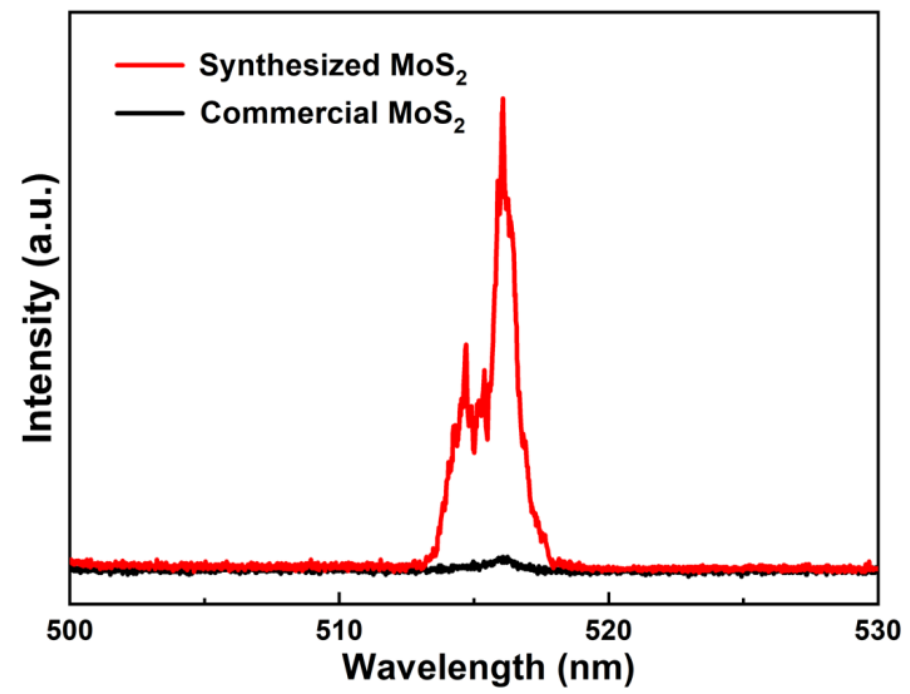

Figure S3. The second harmonic signal of synthesized $\mathrm{MoS}_{2}$ and commercial $\mathrm{MoS}_{2}$.
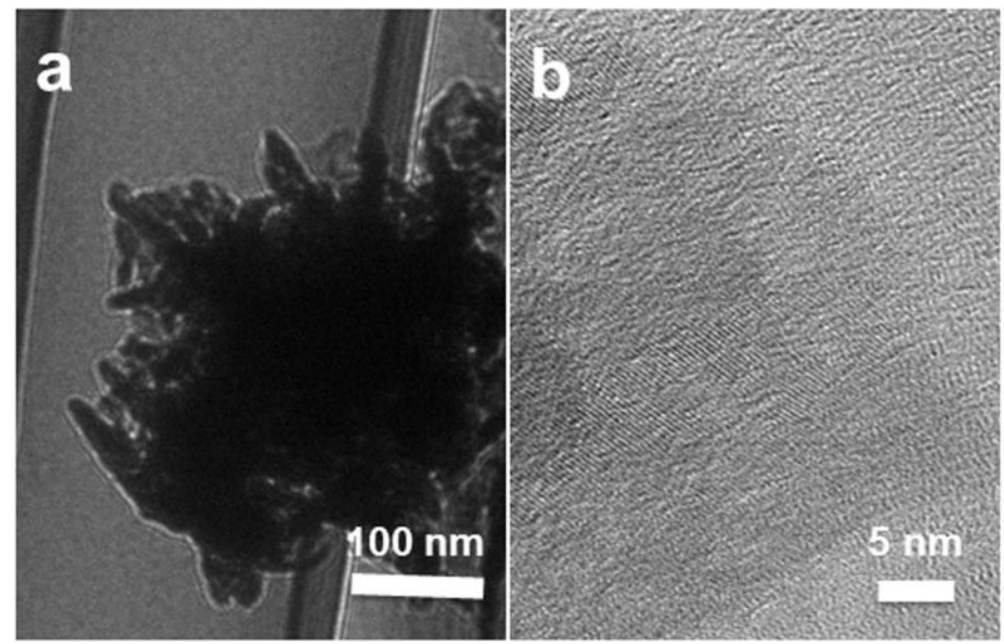

Figure S4. TEM image (a) and HRTEM image (b) of commercial MoS2. 

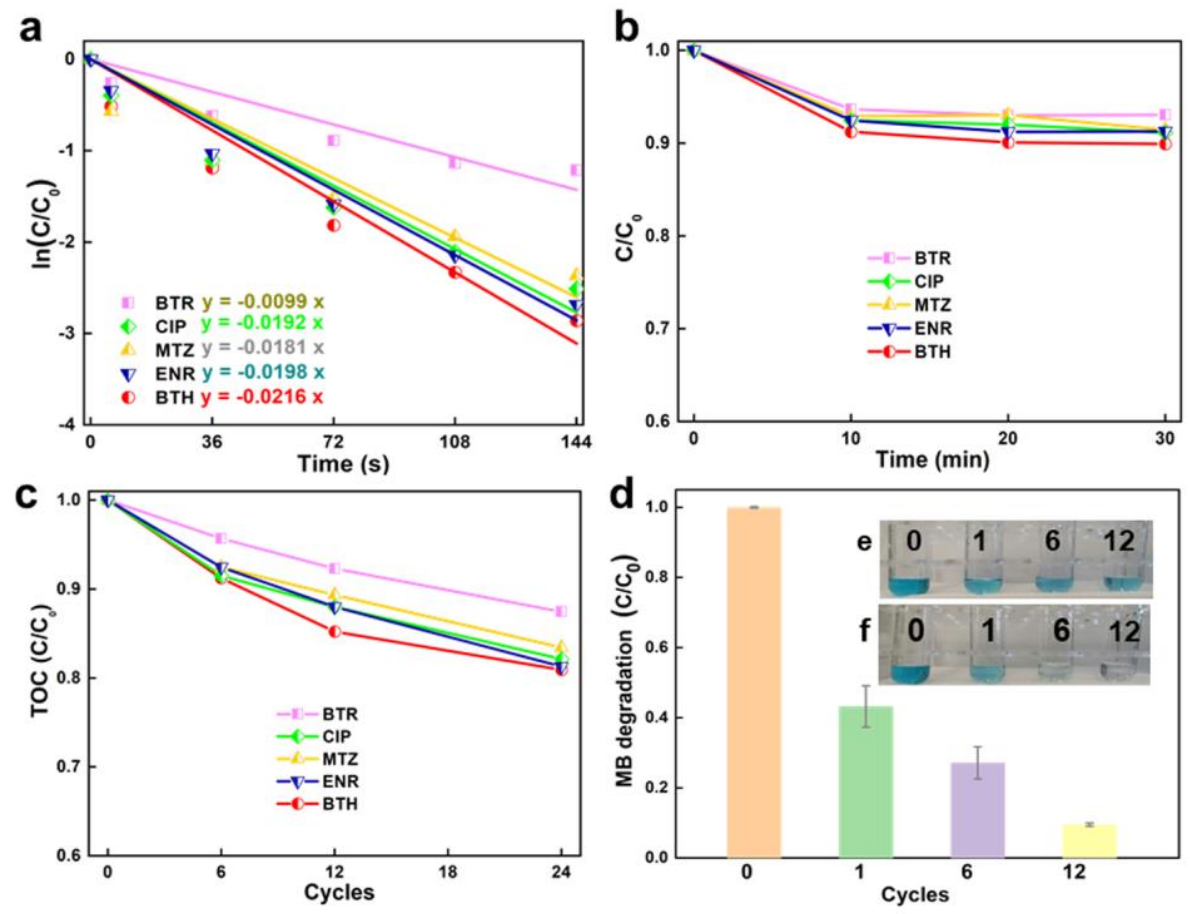

Figure S5. (a) Fitted pseudo-first-order curves of different pollutants in the reactor; (b) adsorption performance of different pollutants in quiescent solution within $30 \mathrm{~min}$; (c) TOC changes of different pollutants in self-powered reactor; (d) concentration changes with $\mathrm{MB}$ after 1, 6 and 12 cycles in the flowing system (Insert: comparison of MB color changes in the quiescent $\mathrm{MoS}_{2}$ experiment (e) and in the flowing suspensions (f)). Experimental conditions: [pollutants $]_{0}=10 \mathrm{mg} / \mathrm{L},\left[\mathrm{MoS}_{2}\right]_{0}=0.5 \mathrm{~g} / \mathrm{L}$, MB (methylene blue). 

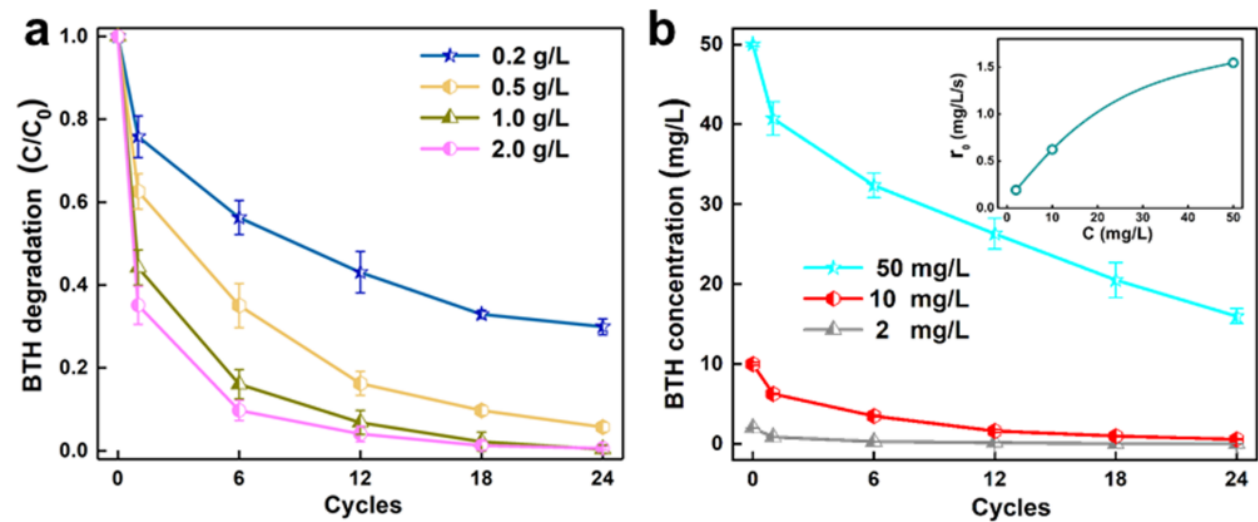

Figure S6. Operational variables influencing the BTH removal: (a) removal efficiency of different catalyst dosage with $10 \mathrm{mg} / \mathrm{L} \mathrm{BTH}$; (b) concentration changes of different initial BTH concentration with $0.5 \mathrm{~g} / \mathrm{L} \mathrm{MoS}_{2}$. (Inset: initial rate vs BTH concentration)
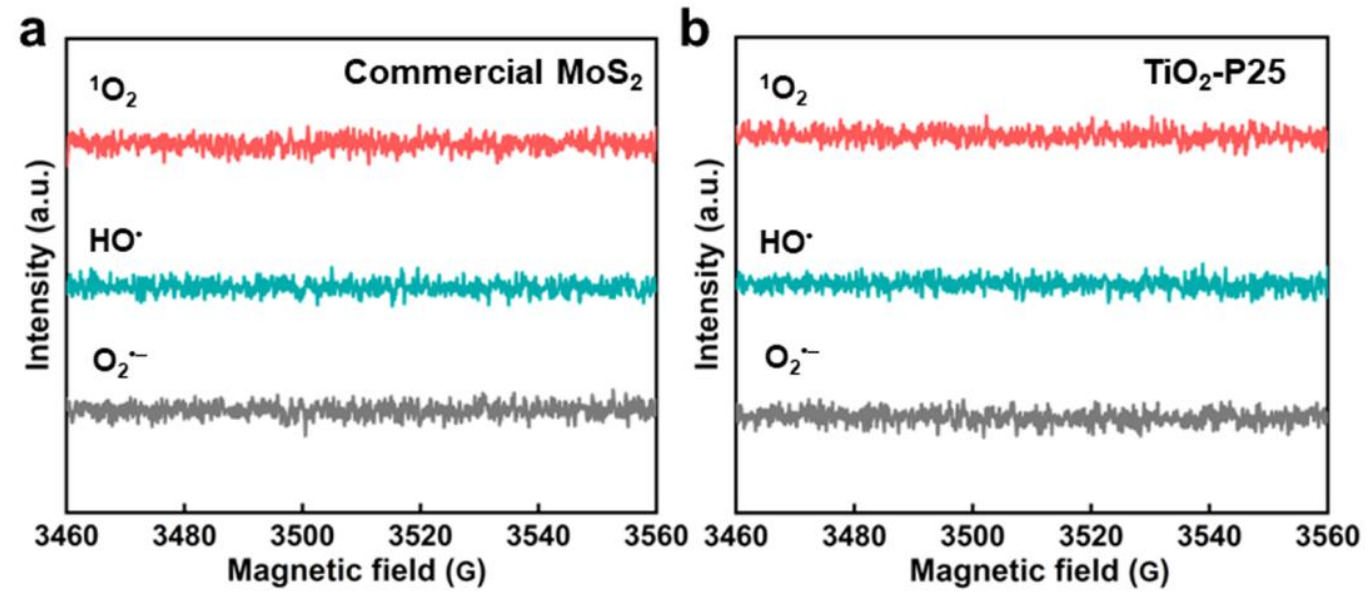

Figure S7. EPR spectra of $\mathrm{O}_{2}{ }^{--}, \mathrm{HO}^{\bullet}$ and ${ }^{1} \mathrm{O}_{2}$ of commercial $\mathrm{MoS}_{2}$ (a) and $\mathrm{TiO}_{2}-\mathrm{P} 25$ (b) in the water flow. 

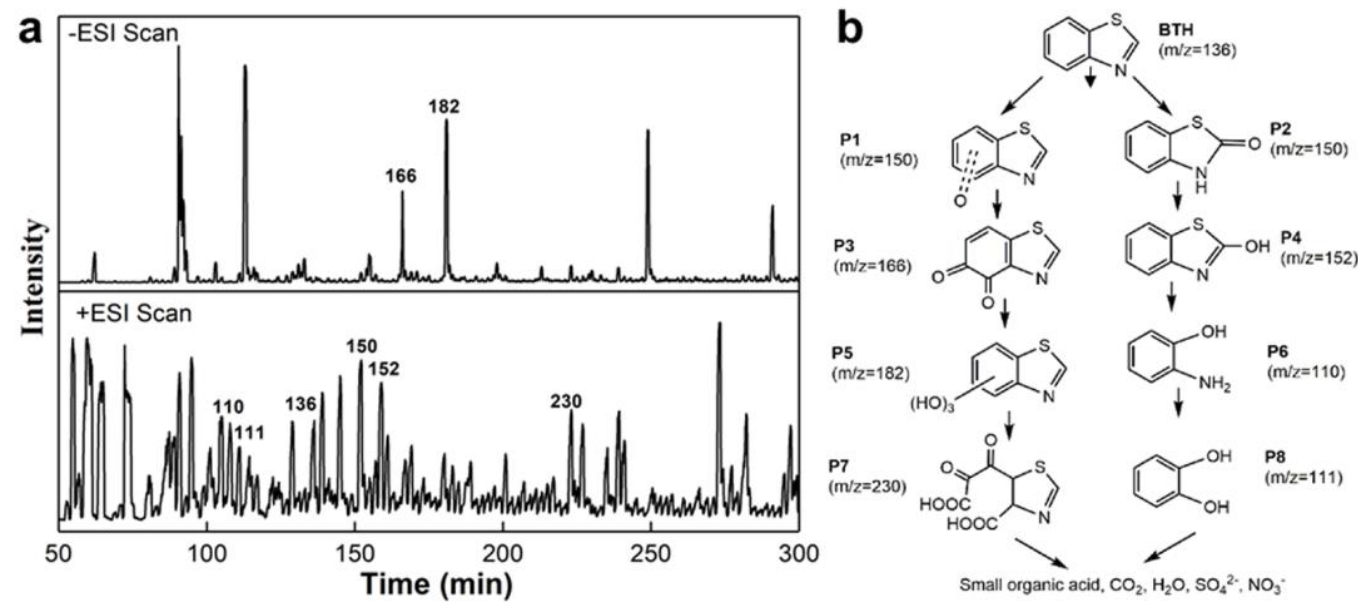

Figure S8. LC-MS analysis and pathways proposed for BTH degradation during $\mathrm{MoS}_{2}$ piezocatalytic process.
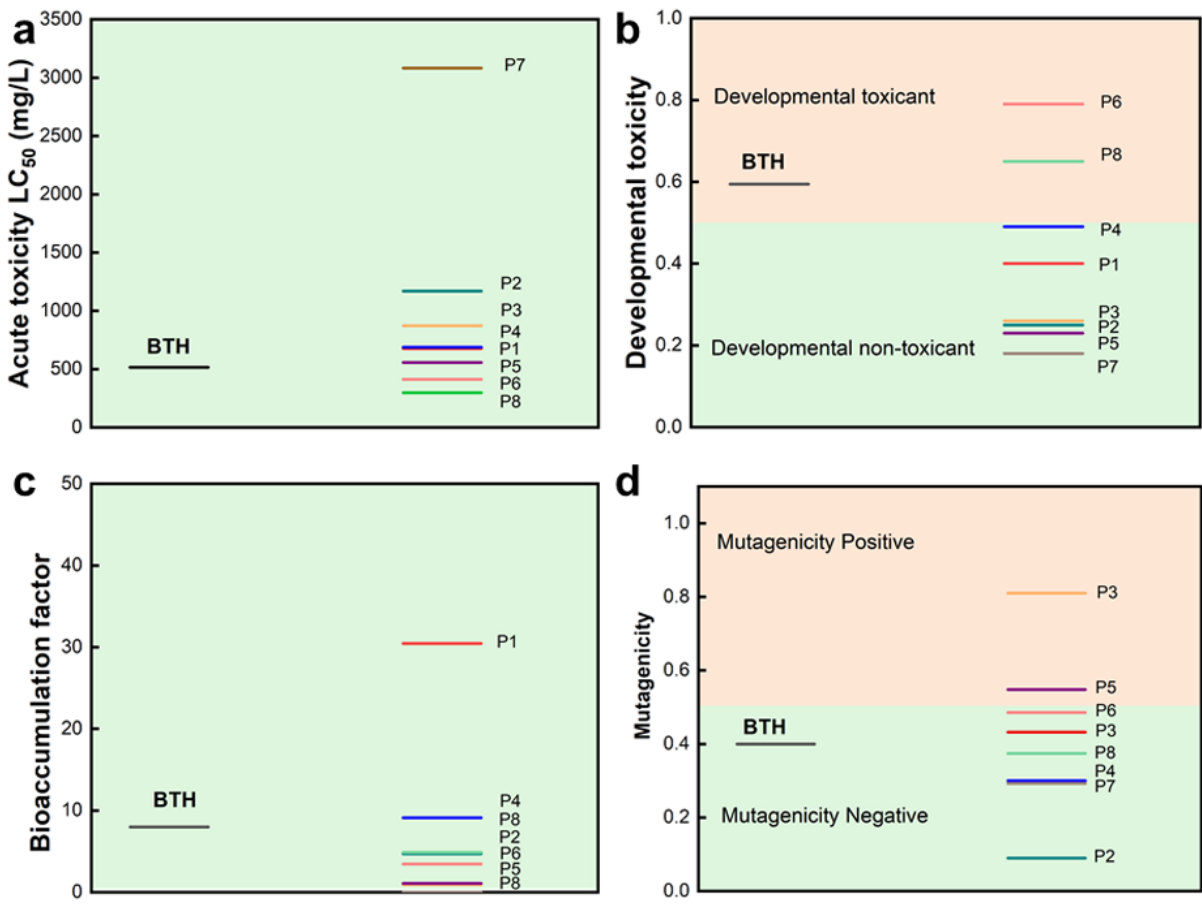

Figure S9. (a) Acute toxicity; (b) developmental toxicity; (c) bioaccumulation factor; (d) mutagenicity of BTH and its degradation products. 


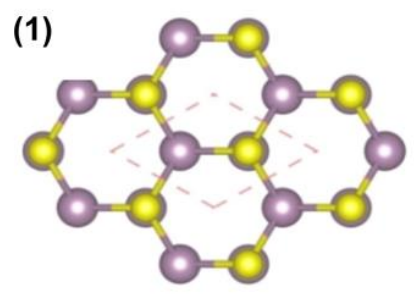

(4)

808080889898089800 कomonomonomo \&.8\%

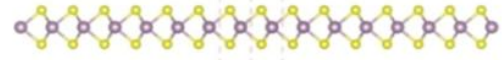
\%8\% 8 \% 8 \% $8 \% 8 \%$
(2)

(3) fopzopozozozo

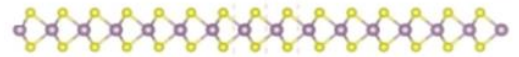

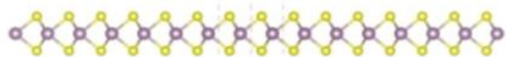

(5)

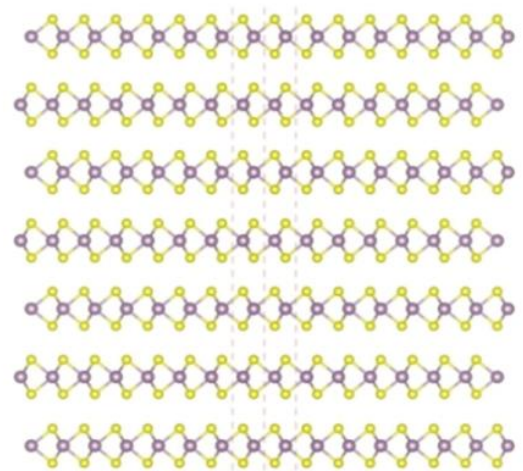

Figure 10. The atomic structure of odd-layered $\mathrm{MoS}_{2}$

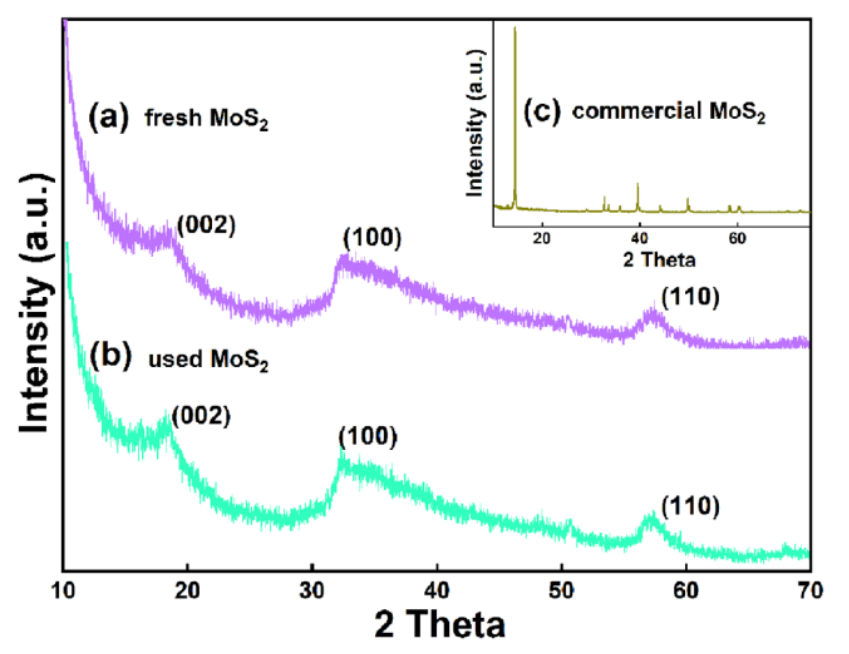

Figure S11. XRD patterns of fresh $\operatorname{MoS}_{2}$ (a) and used $\mathrm{MoS}_{2}(\mathrm{~b})$; (c) XRD pattern of commercial $\mathrm{MoS}_{2}$. 


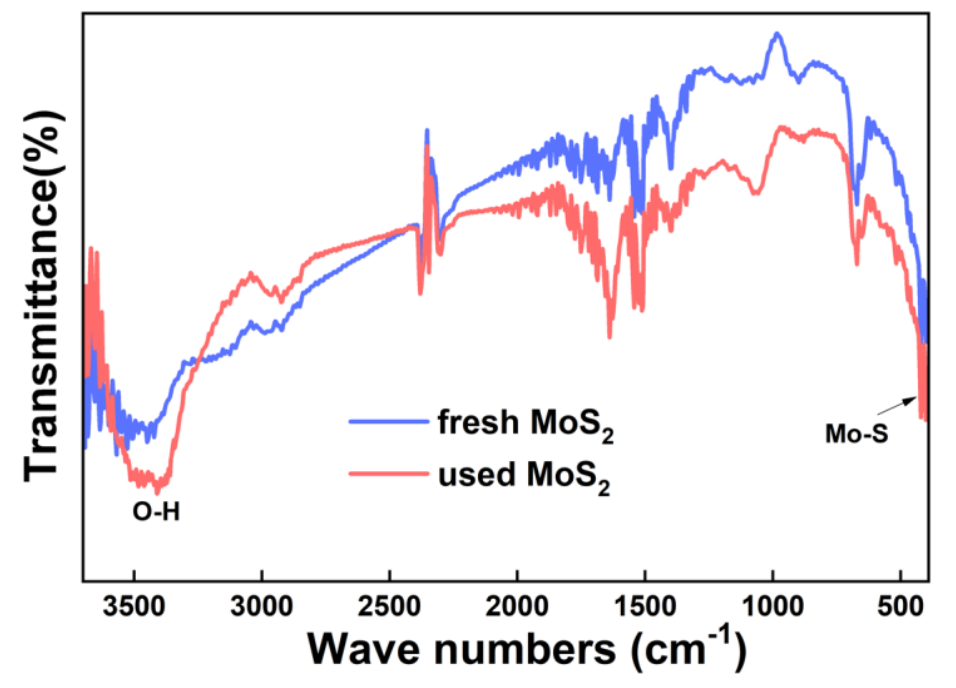

Figure S12. FTIR spectra of $\mathrm{MoS}_{2}$ before and after reaction 


\section{Supporting Tables}

Table S1. Characterizations of the real wastewater matrices

\begin{tabular}{ccccc}
\hline Wastewater & Sewage & Rain & Saline & DIW \\
\hline $\mathrm{pH}$ & 3.2 & 5.5 & 7.0 & 6.7 \\
TOC (mg/L) & 128.2 & 2.2 & ND & ND \\
Turbidity (NTU) & 43.5 & 1.9 & ND & ND \\
\hline
\end{tabular}

